\title{
AULA INVERTIDA CON USO DE RECURSOS TECNOLÓGICOS: SUS EFECTOS SOBRE EL APRENDIZAJE Y LA ACTITUD HACIA LAS MATEMÁTICAS EN UNA MUESTRA DE ESTUDIANTES DE HONDURAS
}

\author{
FLIPPED CLASSROOM WITH THE USE OF \\ TECHNOLOGICAL RESOURCES: ITS EFFECTS ON \\ LEARNING AND ATTITUDE TOWARDS \\ MATHEMATICS IN A SAMPLE OF \\ HONDURAN STUDENTS
}

\author{
José Alberto Núñez Paz \\ Maranatha Adventist Bilingual School, Honduras \\ alberto_44@hotmail.com \\ https://orcid.org/0000-0002-0423-3632 \\ Jaime Rodríguez \\ Instituto Universitario del Sureste, México \\ jar@um.edu.mx \\ https://orcid.org/0000-0003-4761-140X
}

\begin{abstract}
RESUMEN
La tecnología es parte integral del proceso educativo, por lo que es necesario conocer sus efectos sobre el rendimiento y la actitud hacia las matemáticas de los estudiantes. El presente estudio procuró determinar si existe una diferencia significativa de desempeño matemático y de actitud hacia las matemáticas entre un grupo en el que se utilizó la clase invertida con el uso de recursos tecnológicos y uno en el que se utilizó la clase tradicional. Participaron 38 estudiantes del segundo año de Bachillerato en Ciencias y Humanidades en una escuela privada en Honduras, durante el curso escolar 2018-2019. La investigación fue de tipo cuantitativa, con un diseño cuasiexperimental. Se utilizó la prueba estadística no paramétrica $U$ de Mann Whitney para dos muestras independientes. Se encontró un efecto positivo al aplicar la clase invertida con el uso de recursos tecnológicos sobre el rendimiento académico y sobre la actitud hacia las matemáticas en sus cuatro dimensiones: valor, autoconfianza, disfrute y motivación.
\end{abstract}

Palabras clave: tecnología educativa, rendimiento académico, actitud hacia las matemáticas, aula invertida

\section{ABSTRACT}

Technology is an integral part of the educational process, so it is necessary to know its effects on students' performance and attitude towards 
mathematics. The present study sought to determine whether there is a significant difference in mathematical performance and attitude towards mathematics between a group in which the flipped class was used with technological resources and another one in which the traditional class was used. Thirty-eight students of the second year of Baccalaureate in Sciences and Humanities in a private school in Honduras participated in the study during the 2018-2019 school year. The research was quantitative, with a quasi-experimental design. The nonparametric Mann-Whitney U statistical test was used for two independent samples. A positive effect was found when applying the inverted class with the use of technological resources on academic performance and the attitude towards mathematics in its four dimensions: value, self-confidence, enjoyment, and motivation.

Keywords: educational technology, academic performance, attitude towards mathematics, flipped classroom

\section{Introducción}

El avance en la tecnología y su fácil acceso ha creado nuevas metodologías de enseñanza que permiten una mayor comprensión por parte de los estudiantes. En la actualidad, la enseñanza de las matemáticas ha tenido un cambio significativo. Los libros de texto cuentan con plataformas virtuales para ayudar a los estudiantes a una mejor comprensión y las aplicaciones para dispositivos móviles permiten resolver problemas paso a paso.

Los avances tecnológicos y el acceso a la tecnología en todos los ámbitos de la vida hacen necesario introducir tecnologías orientadas a la construcción de conocimientos en los estudiantes para lograr un proceso de enseñanza más eficiente y adaptado a las nuevas necesidades, además de permitir a los alumnos crear su propio conocimiento. Una opción es la clase invertida (flipped classroom), que facilita la aplicación de innovaciones tecnológicas para mejorar el rendimiento escolar (Connor et al., 2014; Strohmyer, 2016). La opción permite que los estudiantes asimilen los conceptos básicos con videos y otros materiales en casa y que desarrollen su comprensión por medio de trabajos en grupos pequeños, para atender las necesidades académicas de una forma más personal en clase (Kinderman, 2015; Ramaglia, 2015).

Según Bergmann y Sams (2015), la clase invertida, aplicada a la clase de matemáticas, no solo mejora el desempeño académico, sino que además incrementa la motivación.

De acuerdo con el Centro Nacional de Educación de Estados Unidos de América (Gray et al., 2010), la tecnología permite a los maestros reforzar y trasmitir conocimiento. También les permite implementar distintas metodologías pedagógicas. En la actualidad han surgido nuevas tecnologías que han tenido un impacto positivo en la educación (Spector, 2013).

Algunos de los problemas existentes en la enseñanza de las matemáticas son la falta de interacción de los estudiantes con el profesor, el bajo interés que muestran los estudiantes y la escasez de ejercicios realizados bajo la supervisión del docente (Ni et al., 2015).

En este sentido se hace una presentación del aula invertida, considerando cómo ha sido implementada y en qué 
áreas ha beneficiado a los estudiantes en la formación de sus conocimientos y a los docentes en el papel de facilitadores. También se hace referencia al uso de las plataformas educativas y cómo estas interactúan en el proceso de enseñanza-aprendizaje, así como al uso de la tecnología como recurso didáctico, tanto dentro como fuera del salón de clase.

\section{La clase invertida}

Una alternativa educativa que se ha puesto en práctica en los últimos años es la clase invertida, la cual consiste en cambiar la forma tradicional de impartir clases, facilitando videos y otros recursos a los estudiantes mediante una plataforma virtual y se utiliza el tiempo de clase para desarrollar ejercicios en grupo atendiendo las necesidades individuales de cada uno (García de Oliveira Fassbinder et al., 2014; Giannakos et al., 2016).

La clase invertida incrementa la capacidad de trabajo autónomo del estudiante al trabajar en casa según sus propias necesidades, así como su autoeficacia y la capacidad para trabajar de forma colaborativa en clase y mejorar su desempeño matemático (Aşiksoy y Özdamli, 2016). Según Morgan (2014), la clase invertida permite a los estudiantes ver varias veces el contenido que les resulta difícil y solo repasar brevemente el contenido fácil, según el criterio de cada estudiante, mientras que en la clase se desarrollan ejercicios y resuelven dudas. Files (2016) observó que la clase invertida es aplicable a las matemáticas y que en álgebra ha dado buenos resultados en comparación con la clase tradicional y la clase en línea. Sin embargo, destacó que la falta de madurez de los estudiantes puede ser un factor negativo para este tipo de metodología. Un factor importante para la educación es el uso de las plataformas adecuadas (Bhagat y Chang, 2015; Bhagat et al., 2016; Puccetti, 2016). Estas plataformas ofrecen herramientas pedagógicas que permiten la visualización de temas abstractos, el desarrollo de lógica matemática, el reforzamiento en cualquier momento, la asignación de tareas y tutoriales especializados que guían al estudiante y mantienen registro de rendimiento, además de la retroalimentación por parte del maestro (Charles, Hall, Kenedy, Bass et al., 2015; Charles, Hall, Kennedy, Bellman et al., 2015; Serway y Faughn, 2012). Las plataformas virtuales representan una facilidad pedagógica para el estudiante al permitirle elegir la metodología adecuada para cumplir con los requisitos académicos que le son exigidos. Es preciso resaltar que tales plataformas constituyen una ayuda pedagógica y no una sustitución de la clase presencial (Arnold-Garza, 2014), ya que durante la clase se resuelven ejercicios, se aclaran dudas y se evalúa de forma inmediata el desempeño de cada estudiante, enfocándose en las necesidades específicas que precise mejorar (Bergmann y Sams, 2012; Lasry et al., 2014).

El uso de la tecnología, además de mejorar la forma de aprendizaje, facilita la forma en que se imparte conocimiento adecuado a los requerimientos actuales (Mau, 2016). Los maestros buscan los recursos que se adecuen a su metodología y objetivo (Huereca, 2015). Los estudiantes que han utilizado plataformas tecnológicas educativas buscan apoyo de videos y otras utilidades que ofrecen, aun cursando una clase totalmente tradicional (Palmer, 2015).

El currículo académico matemático exige enseñar una gran cantidad de temas y mediante la clase invertida se puede cubrir contenido con mayor facilidad, tanto 
para el estudiante al asimilar el contenido, como para el maestro al impartir las clases (Mason et al., 2013).

\section{El aula invertida y el constructivismo}

Un elemento teórico fundamental para argumentar el uso del aula invertida es el constructivismo. Esta filosofía de aprendizaje se basa principalmente en las ideas de Jean Piaget y Lev Vygotsky. Ambos afirmaban que las personas construyen su conocimiento por medio de las experiencias, la interacción social y la colaboración (Powell y Kalina, 2009). Las instituciones educativas están cambiando de un paradigma consistente con brindar una educación a uno consistente con producir conocimiento (Barr y Tagg, 1995). Para lograrlo, Hunter (2015) señala que el aprendizaje activo usa los medios tecnológicos como herramientas para ayudar al estudiante a crear su propio conocimiento siguiendo su propio ritmo. Además, ayuda a modelar problemas de la vida real haciendo el aprendizaje significativo. Según Overbay et al. (2010), el constructivismo necesita tanto de un guía en la enseñanza como de la construcción del conocimiento por parte del estudiante. Para Cobb (1994), la educación constructivista está estrechamente relacionada con el uso de la tecnología. La tecnología brinda autonomía y autoconfianza en los estudiantes, de tal manera que se acelera el proceso del desarrollo de habilidades de pensamiento de orden superior. En esta metodología el profesor es un guía para el descubrimiento del conocimiento (Adams y Burns, 1999). Con una educación mediada por la tecnología, el estudiante desarrolla el análisis a su propio ritmo y facilita el proceso de enseñanza basado en resolución de problemas, además de otros enfoques del aprendizaje
(Herring, 2004).

\section{Actitud hacia las matemáticas}

La actitud hacia las matemáticas "es una predisposición o tendencia por parte de una persona a responder positivamente o negativamente a algún objeto, situación, concepto o persona" (Aiken, 1970, p. 551). Para Cheung (1988), "la actitud se referirá a percepciones afectivamente ajustadas a situaciones en las que la matemática se aprende" (p. 209). Según McLeod (1992), la actitud, los sentimientos y las creencias pertenecen al dominio afectivo. Define actitud como "respuestas afectivas que involucran sentimientos positivos y negativos, de intensidad moderada y razonable estabilidad" (p. 578). DeBellis y Goldin (1997) también la incluyen en el dominio afectivo y agregan a la definición de McLeod el valor que la persona da a las matemáticas.

Para Jovanovic y King (1998) y Zakariya (2017), la actitud hacia las matemáticas es un atributo personal que está relacionado con los sentimientos, los pensamientos y el poder disfrutar las matemáticas, más que con el dominio de la materia. Según McLeod (1992), el dominio afectivo está dividido en tres partes: actitud, sentimientos y creencias. La actitud incluye sentimientos positivos o negativos de razonable intensidad y estabilidad moderada, los cuales son vistos desde dos perspectivas, desde la reacción emocional automática a las matemáticas o desde una actitud pre-existente a una tarea nueva, pero relacionada con una conocida. Las emociones se refieren a los sentimientos positivos o negativos; son intensos y poco estables. Las creencias pueden referirse a las matemáticas como disciplina o a uno mismo en relación con las matemáticas. 
Hannula (2002) separó la actitud hacia las matemáticas en cuatro procesos evaluativos: (a) las emociones que el estudiante experimenta durante el proceso de actividad matemática, (b) las emociones que el estudiante automáticamente asocia con el concepto matemáticas, (c) las evaluaciones de situaciones que el estudiante espera seguir a consecuencia de hacer matemáticas y (d) el valor de las matemáticas relacionado con las metas.

Para el presente estudio, la actitud hacia las matemáticas es el nivel de autoconfianza, valor, disfrute y motivación relativas a las matemáticas. Esta definición es utilizada para el Attitudes Toward Mathematics Instrument (ATMI), elaborado por Tapia (1996).

La actitud hacia las matemáticas también ha sido un factor importante en el rendimiento académico, dado que algunos estudiantes muestran temor o no se sienten con la confianza suficiente para poder aprenderlas. Una de las herramientas que utiliza la clase invertida es el uso de programas computacionales, los cuales han sido útiles y han mostrado tener un efecto positivo sobre la actitud hacia las matemáticas de los estudiantes (Muthulakshmi y Veliappan, 2015). Además, incrementa en los estudiantes un mayor compromiso con la clase, en comparación con una clase tradicional (Ramaglia, 2015), lo que da como resultado que los logros académicos de los estudiantes en la clase de matemáticas mejoren significativamente mediante la clase invertida.

Debido a la relevancia de estrategias innovadoras como el aula invertida y su efecto en la actitud hacia las matemáticas, este estudio tiene como propósito identificar si existe diferencia significativa en el desempeño matemático y la actitud hacia las matemáticas entre un grupo que utiliza la clase invertida y uno que utiliza la clase tradicional.

\section{Método \\ Tipo de investigación}

La investigación fue cuantitativa, correlacional, cuasi experimental y multivariada, con diseño de pretest y postest con grupo control. Se seleccionaron al azar dos grupos, uno de control y otro experimental.

\section{Participantes}

La población estuvo formada por 38 estudiantes del segundo año de Bachillerato en Ciencias y Humanidades de una escuela privada en Honduras. Se tomaron dos grupos conformados dos grupos, A y B, con 18 y 20 estudiantes respectivamente. Para seleccionar los grupos de forma aleatoria, se solicitó a un estudiante del grupo A que, utilizando la calculadora gráfica TI-92, introdujera la función rand (). Dicha función devuelve valores al azar entre cero y uno. Si el valor obtenido al presionar una vez la función era mayor o igual a 0.5 , el grupo A sería el experimental y, en consecuencia, el grupo B el de control. Si el valor obtenido era menor que 0.5 , el grupo A sería el de control y el grupo B el experimental.

\section{Instrumentos}

Actitud hacia las matemáticas. Para medir la actitud hacia las matemáticas se utilizó la escala de Actitud hacia las Matemáticas (ATMI, por sus siglas en inglés). El instrumento consiste en 40 ítems medidos en una escala Likert en un rango de 1 al 5 , donde 1 corresponde a totalmente en desacuerdo y 5 a totalmente de acuerdo. A su vez, los 40 ítems de la escala se subdividen en cuatro subescalas: 
autoconfianza (SC), valor (V), disfrute (E) y motivación (M). En el caso del pretest, los coeficientes de confiabilidad de las escalas resultaron aceptables, todos con valores alfa de Cronbach superiores a .7, donde la actitud general obtuvo un valor de .904; la autoconfianza, de .882; el valor, de .703; el disfrute, de .828; y la motivación, de .709 .

Rendimiento académico. El rendimiento académico fue medido por los exámenes de control, el pretest y el postest. Se utilizó un examen basado en los temas que establece el Currículo Nacional Básico, correspondiente a la primera unidad del primer semestre en el segundo año de Bachillerato en Ciencias y Humanidades elaborado ex profeso. Para determinar el rendimiento académico se utilizó una escala de 0 a 100 , donde 0 fue la nota más baja posible y 100 la nota más alta. Un mayor puntaje se interpreta como un mayor dominio del contenido.

\section{Tratamiento}

La intervención se realizó durante el desarrollo de la primera unidad, correspondiente al primer semestre del año escolar 2018-2019. En el inicio del curso escolar, a ambos grupos se aplicó el instrumento para medir la actitud matemática y el examen de nivel de conocimiento sobre las temáticas que se estudiarían en esa unidad. El examen de rendimiento académico y la prueba de actitud hacia las matemáticas se administraron al finalizar la unidad académica.

El periodo de clase en el grupo experimental estuvo dividido en tres momentos. En la parte introductoria de la clase se dio tiempo para preguntas y comentarios del tema que se dejó como asignación en las plataformas académicas. Posteriormente, se trabajó en gru- pos pequeños donde se realizaron ejercicios de los temas estudiados en casa y en la parte final de la clase se dejaron asignaciones para el siguiente día, ya sea para complementar lo aprendido o para estudiar un tema nuevo. Las asignaciones fueron de tipo conceptual y se desarrollaron ejercicios en el periodo de la clase.

\section{Plataformas}

Los estudiantes realizaron las actividades mediante la plataforma de la editorial Pearson y los videos fueron creados o seleccionados por el docente y divulgados mediante la plataforma www.edpuzzle.com. En la plataforma Edpuzzle se pueden insertar preguntas en cualquier parte del video, tanto con opción de respuesta única o con respuesta breve. Cada video está dividido en 10 partes y la plataforma muestra al docente cuántas veces fue vista por el estudiante cada parte del video. Además, la plataforma le da la opción al docente de que el estudiante tenga que ver el video sin saltar ninguna parte o que esté libre para que el estudiante vea lo que él crea conveniente. Enseguida se muestran algunos ejemplos de actividades.

Actividad 1. Para la enseñanza del tema de gráficas de funciones racionales, las definiciones de asíntotas y cómo poderlas identificar gráficamente, así como la gráfica de una función racional básica, se envió como asignación un video explicativo con ciertas preguntas integradas (Núñez Paz, 2018a). En clase se trabajó con ejercicios acordes al video seleccionado. Además, cada grupo de ejercicios tenía un ejemplo del libro que le sirvió de guía al estudiante para poder resolverlos (ver Figura 1). 


\section{Figura 1}

Ejercicios en el libro de texto sobre gráficas de funciones racionales

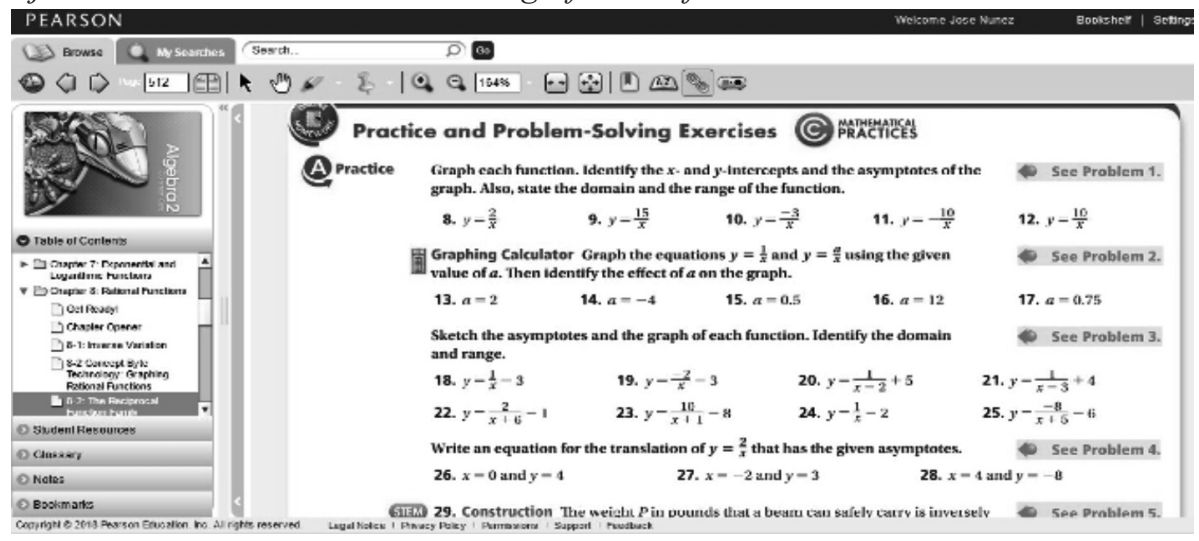

Actividad 2. La actividad 2 tiene que ver con la introducción al tema de límites. Su aproximación a un punto determinado en una función y su concepto de forma gráfica fueron asignados al estudiante mediante un video alojado en la plataforma Edpuzzle
(Núñez Paz, 2018b), con el objetivo de que el estudiante pueda comprender la definición de límite. Se complementó la actividad con una guía evaluativa de conceptos y ejercicios del libro de texto (Blitzer, 2014, p. 1099) (ver Figura 2).

\section{Figura 2}

Ejercicios en el libro de texto sobre el comportamiento de límites

\section{CONCEPT AND VOCABULARY CHECK}

\section{Fill in each blank so that the resulting statement is true.}

1. The notation $\lim _{x \rightarrow} f(x)=L$ is read "the of ${ }_{\text {equals the number }}$ as

2. The notation $\lim f(x)=L$ means that as gets closer to $\longrightarrow$ but remains unequal to the corresponding values of

3. True or false: If $\lim _{x \rightarrow a} f(x)=L$, then the value of $f$ at $a$ is equal to $L$.

4. The notation $\lim _{x \rightarrow f(x)}=L$ is called the limit. The notation means that as

closer to the corresponding values of to
5. The notation $\lim _{x \rightarrow} f(x)=L$ is called the limit. The notation means that as closer to but remains greater than the corresponding values of get closer to

6. If $\lim _{x \rightarrow} f(x)=L$ and $\lim _{x \rightarrow \infty} f(x)=L$, then $\lim f(x)$

7. If $\lim _{x \rightarrow x^{-}} f(x)=L$ and $\lim _{x \rightarrow \infty} f(x)=M$, where $L \neq M$, then $\lim _{x \rightarrow \infty} f(x)$

\section{EXERCISE SET 11.1}

\section{Practice Exercises}

In Exercises I-4, use cach table to find the indicated limit.

$$
\text { 1. } \lim _{x \rightarrow 2} 2 x^{2}
$$

\begin{tabular}{|l|l|l|l|l|l|}
\hline$x$ & 1.99 & 1.999 & $1.9999 \rightarrow \leftarrow 2.0001$ & 2.001 & 2.01
\end{tabular} $f(x)=2 x^{2} 7.9202 \quad 7.9920 \quad 7.9992 \rightarrow \leftarrow 8.0008 \quad 8.0080 \quad 8.0802$ 2. $\lim _{x \rightarrow 3} 5 x^{2}$

\begin{tabular}{|l|l|l|l|l|l|}
\hline$x$ & 2.99 & 2.999 & $29999 \rightarrow-3.0001$ & 3.001 & 3.01 \\
\hline
\end{tabular} \begin{tabular}{l|l|l|l|l|l|l|l|}
$f(x)=5 x^{2}$ & 44.701 & 44.970 & $44.997 \rightarrow \leftarrow 45.003$ & 45.030 & 45.301
\end{tabular}

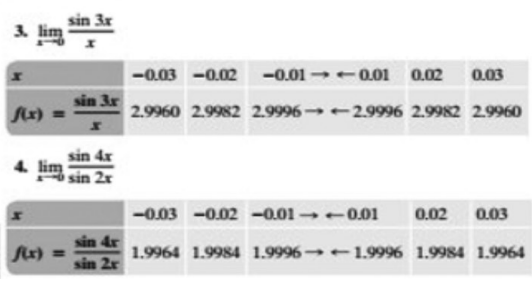


Experiencias de uso de la plataforma Edpuzzle

Los presentes datos fueron obtenidos mediante la utilización de la plataforma Edpuzzle en la clase de matemáticas. En ella es posible llevar un control general de cada estudiante por asignación e indica si el video fue visto. También muestra cuál es el porcentaje de respuestas correctas de las preguntas que están en el video (ver Figura 3).

De forma particular, se brinda por estudiante un detalle de la fecha, los puntos obtenidos y el número de respuestas correctas con base en el total de preguntas. Asimismo, se muestra el porcentaje del video visto, cuántas veces se repitió cada parte del video y cuánto tiempo le tomó completar la asignación (ver Figura 4).

\section{Figura 3}

Estadística general por asignación de la plataforma Edpuzzle

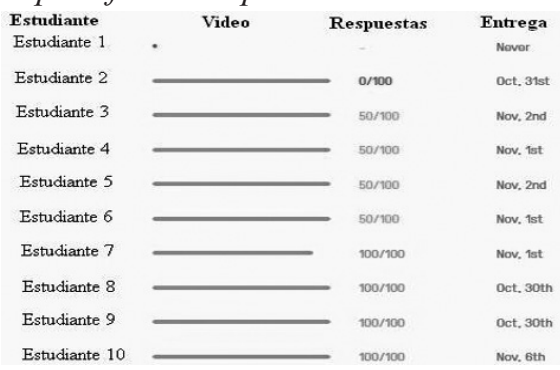

\section{Figura 4}

Estadística por estudiante de cada asignación de la plataforma Edpuzzle

(3) edpuzzle

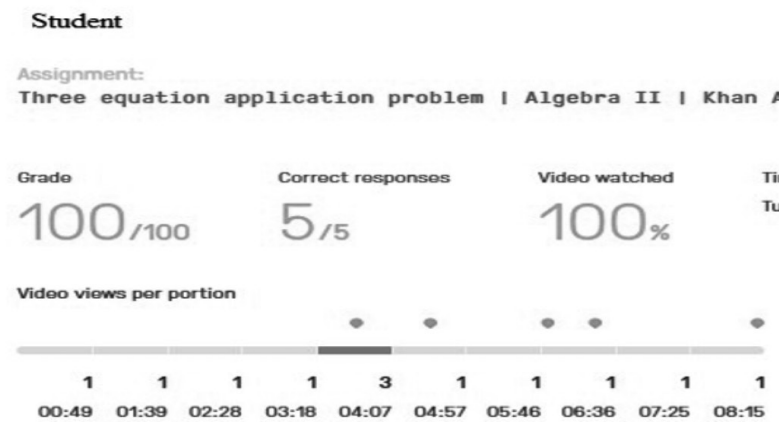

\section{Plataforma Pearsonrealize}

Mediante la plataforma de Pearson, los estudiantes de ambos grupos tuvieron la oportunidad de leer el libro de texto en línea, además de poder ver los ejemplos del libro resueltos por medio de videos explicativos. La plataforma incluso brinda una gama de ejercicios adicionales para los estudiantes que deseen aprender más de lo provisto en clase o repasar lo que ya ha sido enseñado.

\section{Análisis de datos}

Además de hacer análisis descriptivo de las variables involucradas, se utilizó la prueba U Mann-Whitney para muestras independientes.

\section{Análisis descriptivo}

\section{Resultados}

En esta sección se describen las distribuciones de las variables del estudio: actitud hacia las matemáticas y calificaciones de los estudiantes. En 
el caso del pretest, los coeficientes de confiabilidad de las escalas resultaron aceptables, todos con valores alfa de Cronbach superiores a .7. La Tabla 1 contiene los descriptivos del pretest de actitud hacia las matemáticas y sus factores, considerando el grupo control y el grupo experimental. Tanto en el grupo experimental como en el grupo control, se observa que la motivación se ubica en el nivel de acuerdo (valores entre $3.5 \mathrm{y}$ 4.5) únicamente en el factor valor de las matemáticas. En el resto de los factores y de la actitud en general, las respuestas muestran una actitud neutral hacia las matemáticas (valores entre 2.5 y 3.5). Por otro lado, el grupo control tiende a ser más homogéneo, ya que las desviaciones estándar son menores para todos los factores y para la actitud en general.

Tabla 1

Descriptivos para el pretest de actitud hacia las matemáticas según el grupo

\begin{tabular}{lccccc}
\hline & \multicolumn{4}{c}{ Grupo control } & \multicolumn{3}{c}{ Grupo experimental } \\
\cline { 2 - 6 } Factor & $M$ & $D E$ & $M$ & $D E$ & Confiabilidad \\
\hline Actitud general & 3.3 & 0.366 & 3.3 & 0.507 & .904 \\
Valor & 4.1 & 0.372 & 3.9 & 0.399 & .703 \\
Disfrute & 3.1 & 0.638 & 3.2 & 0.652 & .828 \\
Autoconfianza & 3.1 & 0.572 & 3.0 & 0.684 & .882 \\
Motivación & 3.0 & 0.632 & 2.9 & 0.752 & .709 \\
\hline
\end{tabular}

Las confiabilidades en el postest mejoraron de tal forma que hubo una mejor comprensión de los ítems y el alfa de Cronbach resultó mayor a .80 en todas las escalas. La Tabla 2 contiene los descriptivos en el postest de actitud hacia las matemáticas y sus factores, considerando los grupos control y experimental. En el grupo control, el valor se ubica en el nivel de acuerdo (valores entre $3.5 \mathrm{y}$
4.5). En el resto de los factores y de la actitud en general, las respuestas hacen ver una actitud neutral hacia las matemáticas (valores entre 2.5 y 3.5 ). En el grupo experimental, la actitud hacia las matemáticas se ubica en el nivel de acuerdo (valores entre 3.5 y 4.5 ), junto con valor, disfrute y motivación. En este grupo, únicamente la autoconfianza se ubicó en un valor neutral.

\section{Tabla 2}

Descriptivos para el postest de actitud hacia las matemáticas y sus factores según el grupo

\begin{tabular}{lcccccc}
\hline & \multicolumn{7}{c}{ Grupo control } & \multicolumn{2}{c}{ Grupo experimental } & & \\
\cline { 2 - 7 } Factor & $M$ & $D E$ & $M$ & $D E$ & $\alpha$ & $d$ \\
\hline Actitud general & 3.4 & 0.504 & 4.1 & 0.297 & .956 & 1.69 \\
Valor & 4.1 & 0.520 & 4.5 & 0.310 & .833 & 0.93 \\
Disfrute & 3.2 & 0.644 & 4.0 & 0.383 & .844 & 1.51 \\
Autoconfianza & 3.1 & 0.726 & 3.4 & 0.329 & .940 & 0.53 \\
Motivación & 2.9 & 0.684 & 3.9 & 0.491 & .817 & 1.68 \\
\hline
\end{tabular}


El instrumento para medir el rendimiento académico fue un examen basado en los temas que establece el Currículo Nacional Básico para la primera unidad del primer semestre en el segundo año de Bachillerato en Ciencias y Humanidades elaborado ex profeso. El examen está basado en una escala de 0 a 100 puntos. En la Tabla 3 se observa que, en el pretest, las medias se encontraban en valores muy similares, mientras que en el postest la diferencia entre ellas se hace mayor. Al inicio los grupos eran más homogéneos precisamente porque no tenían conocimiento del tema.

\section{Tabla 3}

Descriptivos para el pretest y postest de rendimiento académico según el grupo

\begin{tabular}{cccccc}
\hline & \multicolumn{2}{c}{\begin{tabular}{c} 
Grupo \\
\multicolumn{2}{c}{ Grupo } \\
Test
\end{tabular}} & \multicolumn{2}{c}{ control } & \multicolumn{3}{c}{ experimental } \\
& $M$ & $D E$ & $M$ & $D E$ & $d$ \\
Pretest & 4.6 & 2.355 & 4.9 & 2.121 & \\
Postest & 74.9 & 9.097 & 92.3 & 5.465 & 2.3 \\
\hline
\end{tabular}

\section{Análisis correlacional}

Debido a que la cantidad de sujetos era menor a 30 , se recurrió a la prueba estadística no paramétrica $U$ de Mann-Whitney para dos muestras independientes, obteniendo como resultado una probabilidad menor a $.05(U=$ $31.000, p=.000)$. Esto indica que existe diferencia significativa de calificaciones del postest entre el grupo experimental (donde se usó la clase invertida) y el grupo control. El rango promedio para el grupo experimental fue de 26.4 y para el grupo control de 11.2. El tamaño del efecto para el rendimiento académico, según la $d$ de Cohen $(d=2.3)$, muestra que la diferencia entre el grupo control
$(M=74.9, D E=9.097)$ y el grupo experimental $(M=92.3, D E=5.465)$ es importante. Para eliminar el efecto posible del grupo seleccionado como control se realizó una prueba de diferencia entre los grupos en el pretest. Según la prueba U de MannWhitney $(U=155.000, p=$ .641), no existe evidencia suficiente para rechazar la igualdad entre los grupos. Por lo tanto, las condiciones iniciales de conocimiento entre los grupos, eran similares. De hecho, los rangos promedio son de 19.8 para el grupo experimental y 18.1 para el grupo control.

Por otro lado, según la prueba $U$ de Mann-Whitney, existe una diferencia significativa entre el postest de actitud hacia las matemáticas del grupo que utiliza la clase invertida y el grupo que utiliza la clase tradicional $(U=32.500$, $p=.000$ ). Por lo tanto, con base en los rangos promedios de 27.2 para el grupo experimental y 11.7 para el grupo control, se establece que la actitud hacia las matemáticas resulta significativamente más alta en el grupo experimental que en el grupo control. Cabe aclarar que, al inicio del experimento, los grupos no mostraban diferencias significativas $(U=146.000, p=.325)$. Inclusive el rango promedio del grupo experimental $($ rango $=21.3)$ fue superior, aunque no significativamente, al del grupo control $($ rango $=17.7)$. La diferencia de actitud en general, en el postest, entre el grupo experimental y el grupo control fue muy importante según el tamaño del efecto $(d=1.69)$. Algo similar se observó en las dimensiones de valor $(d=$ $0.934)$, disfrute $(d=1.510)$ y motivación $(d=1.680)$. En la autoconfianza, el tamaño del efecto $(d=0.53)$ muestra una mejoría moderada. En cada una de las dimensiones del postest sobre la actitud hacia las matemáticas, los rangos 
promedios fueron significativamente mayores en el grupo experimental que en el grupo control, como se presenta en la Tabla 4.

\section{Discusión}

Los resultados del presente estudio muestran que la implementación de la clase invertida tiene un efecto positi- vo en el rendimiento académico y en la actitud hacia las matemáticas. Estos resultados concuerdan con los hallazgos encontrados por Hake (1998), quien comparó un grupo que recibe enseñanza tradicional con un grupo donde se utilizaban métodos interactivos, siendo estos últimos la base para el aula invertida.

\section{Tabla 4}

Diferencias de medias entre grupos en las dimensiones de la actitud hacia las matemáticas

\begin{tabular}{lccc}
\hline \multicolumn{1}{c}{ Factor } & $\begin{array}{c}\text { Grupo } \\
\text { control }\end{array}$ & $\begin{array}{c}\text { Grupo } \\
\text { experimental }\end{array}$ & $\begin{array}{c}\text { Significatividad de prueba } \\
\text { Mann-Whitney }\end{array}$ \\
\hline Valor & 14.3 & 24.68 & .003 \\
Disfrute & 12.3 & 26.70 & .000 \\
Autoconfianza & 12.3 & 26.70 & .000 \\
Motivación & 11.9 & 27.10 & .000 \\
\hline
\end{tabular}

Además, Hake encontró una media de aprendizaje superior en dos desviaciones estándar a favor del grupo experimental. Estos resultados también coinciden con los descubrimientos de Crouch y Mazur (2001), quienes observaron que los grupos que recibieron instrucción para trabajar de forma colaborativa tuvieron un incremento en desviaciones estándar de 0.49 a 0.74 durante ocho años de clases en la Universidad de Harvard.

Más recientemente, Deslaurióers et al. (2011), al comparar dos secciones grandes, una de 267 integrantes pertenecientes al grupo control y otra de 271 pertenecientes al grupo experimental, en un curso introductorio de física usando aula invertida, hallaron que el aprendizaje fue mayor al doble en el grupo experimental que en el grupo control. Un resultado con efecto más bajo fue observado por Freeman et al. (2014) en un estudio con 225 estudiantes, 158 con una metodología activa y 67 con una metodología tradicional, donde el grupo con metodología activa tuvo un incremento, en su promedio académico, superior al otro grupo en aproximadamente el 6\%.

Lai y Hwang (2016) realizaron un experimento similar al efectuado en esta investigación con dos grupos de cuarto grado en la clase de matemáticas, un grupo experimental con 20 estudiantes a los cuales se les instruyó con clase invertida y un grupo control con 24 estudiantes a los cuales se les enseñó con clase tradicional. Los resultados del estudio muestran que los estudiantes pertenecientes al grupo experimental con bajo rendimiento académico y baja actitud hacia las matemáticas tuvieron un incremento significativo en las calificaciones, en comparación con el grupo control.

De acuerdo con los resultados observados por otros investigadores, en estudios de temáticas similares, se puede inferir que el resultado de esta investigación 


\section{AULA INVERTIDA EN MATEMÁTICAS}

está acorde con la investigación actual sobre el tema. Es decir, el beneficio del aula invertida, al menos en términos de aprendizaje y actitud, es muy importante, pues brinda una oportunidad al estudiante para que desarrolle su conocimiento y habilidades de aprendizaje. Al parecer, este beneficio se da principalmente por el cambio de la actividad que se realiza en el aula, de un aprendizaje pasivo a un aprendizaje colaborativo e interactivo.

Es importante señalar también la ne- cesidad que existe de capacitar a los docentes para implementar la metodología de clase invertida en el área de matemáticas, cumpliendo con la malla curricular establecida y centrándose en el aprendizaje significativo. Además, es importante realizar un análisis de cómo se está desarrollando la clase durante el período escolar. Finalmente, cabe resaltar que la comunicación con los padres también es determinante para la aplicación y eficacia de esta metodología, especialmente en el seguimiento a los videos vistos en casa.

\section{Referencias}

Adams, S. y Burns, M. (1999). Connecting student learning and technology. Southwest Educational Development Laboratory.

Aiken, L. R. (1970). Attitudes toward mathematics. Review of Educational Research, 40(4), 551-596. https://doi.org/10.3102/00346543040004551

Arnold-Garza, S. (2014). The flipped classroom teaching model and its use for information literacy instruction. Communications in Information Literacy, 8(1), 7-22. https://oi.org/10.13016/M2R040

Aşiksoy, G. y Özdamli, F. (2016). Flipped classroom adapted to the ARCS model of motivation and applied to a physics course. Eurasia Journal of Mathematics, Science and Technology Education, 12(6), 1589-1603. https://doi.org/10.12973/eurasia.2016.1251a

Barr, R. B. y Tagg, J. (1995). From teaching to learning: A new paradigm for undergraduate education. Change: The Magazine of Higher Learning, 27(6), 13-26. https://doi.org/10.1080/00091383.1995.10 544672

Bergmann, J. y Sams, A. (2012). Flip your classroom: Reach every student in every class every day. International Society for Technology in Education.

Bergmann, J. y Sams, A. (2015). Flipped learning for math instruction. International Society for Technology in Education.

Bhagat, K. K. y Chang, C. Y. (2015). Incorporating GeoGebra into geometry learning: A lesson from India. Eurasia Journal of Mathematics, Science and Technology Education, 11(1), 77-86. https://oi. org/10.12973/eurasia.2015.1307a

Bhagat, K. K., Chang, C. N y Chang, C. Y. (2016). The impact of the flipped classroom on mathematics concept learning in high school. Journal of Educational Technology and Society, 19(3), 134-142. https://www.jstor.org/stable/10.2307/jeductechsoci.19.3.134

Blitzer, R. (2014). Precalculus (5a ed.). Pearson.

Charles, R. I., Hall, B., Kennedy, D., Bass, L. E., Jonson, A., Murphy, S. J, y Wiggins, G. (2015). Geometry Common Core. Pearson.

Charles, R. I., Hall, B., Kennedy, D., Bellman, A. E., Bragg, S. C., Handlin, W. G., Murphy, S. J. y Wiggins, G. (2015). Algebra 2 Common Core. Pearson.

Cheung, K. C. (1988). Outcomes of schooling: Mathematics achievement and attitudes towards mathematics learning in Hong Kong. Educational Studies in Mathematics, 19(2), 209-219. https:// doi.org/10.1007/978-94-017-2209-4_6 


\section{NÚÑEZ PAZ Y RODRÍGUEZ}

Connor, K. A., Newman, D. L y Deyoe, M. M. (2014, 15-18 de junio). Flipping a classroom: A continual process of refinement [Ponencia]. ASEE 121st Anual Conference and Exposition, Indianapolis, IN, Estados Unidos.

Cobb, P. (1994). Constructivism in mathematics and science education. Educational Researcher, 23(7), 4. https://doi.org/10.3102/0013189X023007004

Crouch, C. H. y Mazur, E. (2001). Peer instruction: Ten years of experience and results. American Journal of Physics, 69(9), 970-977. https://doi.org/10.1119/1.1374249

DeBellis, V. A. y Goldin, G. A. (1997). The affective domain in mathematical problema solving. En S. Benson et al. (Eds.), Proceedings of the Twenty-First International Conference for the Psychology of Mathematics Education (Vol. 2, pp. 209-216). University of Helsinki, Department of Teacher Education.

Deslauriers, L., Schelew, E. y Wieman, C. (2011). Improved learning in a large-enrollment physics class. Science, 332(6031), 862-864. https://doi.org/10.1126/science.1201783

Files, D. D. (2016). Instructional approach and mathematics achievement: An investigation of traditional, online, and flipped classrooms in college algebra [Tesis doctoral, Florida Institute of Technology]. DSpace. https://repository.lib.fit.edu/handle/11141/834

Freeman, S., Eddy, S. L., McDonough, M., Smith, M. K., Okoroafor, N., Jordt, H. y Wenderoth, M. P. (2014). Active learning increases student performance in science, engineering, and mathematics. Proceedings of the National Academy of Sciences, 111(23), 8410-8415. https://doi.org/10.1073/ pnas. 1319030111

García de Oliveira Fassbinder, A., Moreira, D., Cruz, G. y Barbosa, E. F. (2014). Tools for flipped classroom model: An experiment in teacher education. En 2014 IEEE Frontiers in Education Conference (FIE) Proceeding. https://doi.org/10.1109/FIE.2014.7044074

Giannakos, M, N., Krogstie, J. y Aalberg, T. (2016). Video-based learning ecosystem to support active learning: Application to an introductory computer science course. Smart Learning Environments, 3(11). https://doi.org/10.1186/s40561-016-0036-0

Gray, L., Thomas, N. y Lewis, L. (2010). Teachers' use of educational technology in US public schools: 2009. First look. National Center for Education Statistics.

Hake, R. R. (1998). Interactive-engagement versus traditional methods: A six thousand student survey of mechanics test data for introductory physics courses. American Journal of Physics, 66(1), 64-74. https://doi.org/10.1119/1.18809

Hannula, M. S. (2002). Attitude towards mathematics: Emotions, expectations and values. Educational Studies in Mathematics, 49(1), 25-46. https://doi.org/10.1023/A:1016048823497

Herring, M. C. (2004). Development of constructivist-based distance learning environments: A knowledge base for K-12 teachers. Quarterly Review of Distance Education, 5(4), 231-242.

Huereca, K. (2015). High school mathematics teachers' connective knowledge of the challenges and possibilities in implementing the flipped learning model: An embedded mixed-methods study (Publicación $N^{\circ}$ 3715436) [Tesis doctoral, Universidad de Texas at El Paso]. ProQuest Dissertations and Theses Global.

Hunter, B. (2015). Teaching for engagement: Part 1: Constructivist principles, casebased teaching, and active learning. College Quarterly, 18(2), 1-16. http://collegequarterly.ca/2015-vol18-num02-spring/hunter.html

Jovanovic, J. y King, S. S. (1998). Boys and girls in the performance-based science classroom: Who's doing the performing? American Educational Research Journal, 35(3), 477-496. https://doi. org $/ 10.3102 / 00028312035003477$

Kinderman, K. A. (2015). The flipped classroom: An alternative to teaching models in an elementary classroom (Publicación № 1767791906) [Tesis de maestría, The University of the Arts]. ProQuest Dissertations and Theses Global. 


\section{AULA INVERTIDA EN MATEMÁTICAS}

Lai, C. L. y Hwang, G. J. (2016). A self-regulated flipped classroom approach to improving students' learning performance in a mathematics course. Computers and Education, 100, 126-140. https://doi. org/10.1016/j.compedu.2016.05.006

Lasry, N., Dugdale, M. y Charles, E. (2014). Just in time to flip your classroom. Physics Teacher, 52(1), 34-37. https://oi.org/10.1119/1.4849151

Mason, G. S., Shuman, T. R. y Cook, K. E. (2013). Comparing the effectiveness of an inverted classroom to a traditional classroom in an upper-division engineering course. IEEE Transactions on Education, 56(4), 430-435. https://doi.org/10.1109/TE.2013.2249066

Mau, D. (2016). A case study of middle schools teachers' perceptions of the use of classroom websites [Tesis doctoral, Walden University]. Walden Dissertations and Doctoral Studies. https://scholarworks. waldenu.edu/dissertations/2218

McLeod, D. B. (1992). Research on affect in mathematics education: A reconceptualization. En D. A. Grouws (Ed.), Handbook of research on mathematics teaching and learning (pp. 575-596). Macmillan.

Morgan, H. (2014). Focus on technology: Flip your classroom to increase academic achievement. Childhood Education, 90(3), 239-241. https://doi.org/10.1080/00094056.2014.912076

Muthulakshmi, P. y Veliappan, A. (2015). Effectiveness of an interactive multimedia learning package in developing attitude towards mathematics. Journal on School Educational Technology, 11(3), 40-46. https://doi.org/10.26634/jsch.11.3.4789

Ni, M., Lam-for, K., Zhen, L., Xie, Y., Long, H., Zheng, X. y Li, W. (2015). A study of an e-schoolbag supporting flipped classroom model for junior mathematics review class. En S. K. S. Cheung, L. Kwok, H. Yang, J. Fong y R. Kwan (Eds.), Hybrid learning: Innovation in educational practices (pp. 243-254). Springer-Verlag. https://doi.org/10.1007/978-3-319-20621-9_20

Núñez Paz, J. A. (2018a). Section 8.2 Reciprocal function family (edpuzzle). https://edpuzzle.com/ media/5b50df386571113e5e256422

Núñez Paz, J. A. (2018b). Calculus - What is a one sided limit (edpuzzle). https://edpuzzle.com/ media/5b51269ce70eb94118be2a74

Overbay, A., Patterson, A. S., Vasu, E. S. y Grable, L. L (2010). Constructivism and technology use: Findings from the Impacting Leadership Project. Educational Media International, 47(2), 103-120. https://doi.org/10.1080/09523987.2010.492675

Palmer, K. (2015). Massive open online courses: Evaluation and usage patterns of residential students in higher education (Publicación № 1727612911) [Tesis doctoral, Fielding Graduate University]. ProQuest Dissertations and Theses Global.

Puccetti, G. P. (2016). Blended technology rich instruction verses blended computer managed instruction in 8th grade digital literacy instruction (Publicación № 10149966) [Tesis doctoral, Northcentral University]. ProQuest Dissertations and Theses Global.

Powell, K. C. y Kalina, C. J. (2009). Cognitive and social constructivism: Developing tools for an effective classroom. Education, 130(2), 241-251.

Ramaglia, H. (2015). The flipped mathematics classroom: A mixed methods study examining achievement, active learning, and perception (Publicación No 1761168648) Tesis doctoral, Kansas State University]. ProQuest Dissertations and Theses Global.

Serway, R. A y Faughn, J. S. (2012). Physics. Holt McDougal.

Spector, J. M. (2013). Emerging educational technologies and research directions. Educational Technology and Society, 16(2), 21-30. https://www.ds.unipi.gr/et\&s/journals/16_2/3.pdf

Strohmyer, D. A. (2016). Student perceptions of flipped learning in a high school math classroom [Tesis doctoral, Walden University]. Walden Dissertations and Doctoral Studies. https://scholarworks. waldenu.edu/cgi/viewcontent.cgi?article=3281\&context=dissertations 


\section{NÚÑEZ PAZ Y RODRÍGUEZ}

Tapia, M. (1996, 6-8 de noviembre). The Attitudes Toward Mathematics Instrument [Presentación de documento]. Reunión anual de la Mid-South Educational Research Association, Tuscaloosa, AL, Estados Unidos.

Zakariya, Y. F. (2017). Development of Attitudes towards Mathematics Scale (ATMS) using Nigerian data - Factor analysis as a determinant of attitude subcategories. International Journal of Progressive Education, 13(2), 74-84. https://ijpe.penpublishing.net/makale/237

Recibido: 13 de junio de 2020

Revisado: 2 de julio de 2020

Aceptado: 24 de julio de 2020 